\title{
Abandon gastric lavage in the accident and emergency department?
}

\author{
A. T. PROUDFOOT \\ Regional Poisoning Treatment Centre, Royal Infirmary, Edinburgh
}

The very idea is bound to provoke reactions varying from 'not before time' at one extreme to open-mouthed incredulity at the other. However, any response is likely to be based on custom and preconceptions rather than objective assessment of the value of lavage, if only because the evidence is very limited and its interpretation fraught with difficulties. Gastric lavage has been a popular method of emptying the stomach for many years and is still recommended by a number of recent textbooks on poisoning (Matthew \& Lawson, 1979; Proudfoot, 1982; Vale \& Meredith, 1981). To advocate abandoning it is to attack one of the very pillars of the management of poisoning by ingestion and cannot be supported lightly. However, endorsement by common usage should not blind physicians to its limitations or protect it from critical appraisal.

\section{Historical perspective}

Emesis seems to have been the method of emptying the stomach until Jukes (1822) described his 'exhausting pump' and Bush (1822) his 'gastric exhauster' in the London Medical and Physical Journal. It is perhaps worth noting that the stimulus to these surgeons to devise equipment for gastric aspiration and lavage was poisoning with substances such as opium which were thought to render the stomach insensitive to emetics.

In later years lavage seems to have become the method of choice for emptying the stomach in all but first-aid situations. Its hey-day was probably in the 1950 s and 60 sthe barbiturate era and the dawn of the 'physiological' approach to management of hypnotic poisoning pioneered by the Scandinavians. Most objective studies of the efficacy of gastric lavage were carried out, mainly on patients with barbiturate poisoning, about this time and it has received little active attention since. However, the pattern of poisoning now is radically different from that in the 1960 s, far less than in 1822 (Proudfoot \& Park, 1978). Today, opium poisoning by ingestion is a rarity and although narcotic ingestion in the form of dextropropoxyphene/paracetamol analgesics

Correspondence: $\operatorname{Dr}$ A. T. Proudfoot, Regional Poisoning Treatment Centre, Royal Infirmary, Lauriston Place, Edinburgh, EH3 $9 Y W$ 
is common, the importance of gastric emptying in management has been greatly reduced by the availability of naloxone, a potent narcotic antagonist. Moreovero barbiturate poisoning is in rapid decline and the relevance of studies of lavage in this type of poisoning to more modern drugs is open to question. Tricyclic antidepressants? are now by far the most common and important CNS depressants encountered in acutes poisoning but the incidence of overdosage with them has been fairly static in recent years and is highly improbable that they will ever dominate the overdose scene in the way that barbiturates did in the 1960s. Benzodiazepines have replaced barbiturates as sedatives and hypnotics and are currently involved in about $45 \%$ of all self-poisoning episodes. The remarkable feature of these drugs is their safety margin, even in ${ }^{\rho}$ overdoses of $80-100$ tablets-it is so huge that Dr Henry Matthew was prompted to $\vec{P}$ state that the only way one could kill a mouse with Mogadon was to smother it under $\vec{a}$ heap of the tablets! Not surprisingly the changes in drugs used for self-poisoning have had a marked influence on the resulting physical morbidity and overall taking an overdose is now a much safer activity than 20 years ago (Proudfoot \& Park, 1978) $\vec{\sim}$ Clearly the role of gastric lavage requires reassessment in the light of these develop-i ments.

Paediatricians probably led the trend away from gastric lavage. But their's is a special case-there are clearly difficulties in passing tubes large enough to allow recovery of food and tablets in small patients. However in North America, even in adult medicine the pendulum has swung away from lavage to the use of emetics (Haddad \& Winchester $\overrightarrow{x O}$ 1983). To date, British practice has shown little sign of changing similarly.

Before advocating abandonment of gastric lavage it is pertinent to consider whether the changing pattern of poisoning has rendered gastric emptying (by any metho⿳亠 obsolete, its limitations and hazards and whether it really recovers poisons.

\section{Is gastric emptying, by any means, obsolete?}

This question should be raised even if only to be dismissed immediately. For any doctor who has even the most casual contact with emergency care it is superfluous and the answer self-evident. Acute poisoning is second only to ischaemic heart disease as a cause of emergency admissions to medical units and that does not take account of serious. drunkenness. Many potentially lethal drugs and poisons are regularly consumed in overdosage and there are still considerable numbers of deaths from poisoning each yea $\dot{B}$ (Vale \& Meredith, 1981). That some method of emptying the stomach is necessary, and likely to remain so for the forseeable future, is beyond doubt. Common sense dictates that it is prudent to try to retrieve as much poison as possible before it passes through the pylorus to reach the absorptive surface of the jejunum in the hope that morbidity and mortality will be reduced as far as possible. Indeed, failure to empty the stomach in many cases could, rightly in my view, be construed as negligent. While there may be् arguments about how best to do it in conscious patients, in those who are unconscious there is no alternative to gastric lavage.

\section{The limitations and hazards of gastric lavage}

Most of the limitations of gastric lavage are obvious and one of the most important i 
that it is usually incomplete. Almost certainly, not all the drug in the stomach is retrieved by apparently thorough lavage and in extreme cases drug concretions may be found in the stomach at post-mortem examination (Sharman et al., 1975; Schwartz, 1976; Victor et al., 1968). More commonly, some drug escapes recovery by being trapped in mucosal folds. Even more of a limitation is that poison may be flushed through the pylorus by lavage fluid with subsequent increases in plasma concentrations and toxicity. About $25 \%$ of patients with salicylate poisoning show evidence of continued absorption after admission to hospital and gastric lavage (Lawson et al., 1969) and the same almost certainly occurs with other drugs. The possibility that this drawback could be prevented by administration of activated charcoal at the end of the procedure requires assessment.

Moreover, gastric lavage cannot be used indiscriminately. Aspiration of gastric contents during lavage must be one of the commonest hazards, and lavage is clearly contraindicated in unconscious patients unless the airway can be protected by a vigorous cough reflex or by inserting a cuffed endotracheal tube. It is also inadvisable when long-chain hydrocarbons such as petrol, paraffin, paint thinner and numerous other related products (petroleum distillates) are consumed. However, even in this situation if the amount ingested is thought to be sufficient to cause serious CNS toxicity it may be permissible provided the lungs are protected. Similarly the ingestion of corrosives is no more than a relative contraindication to the passage of gastric tubes. Some of these poisons not only cause oesophageal and gastric necrosis but also systemic toxicity if absorbed. Cautious lavage to prevent absorption of toxic radicals may be important and possible if carried out within a short time of ingestion before burns become deep enough to increase the risk of perforation.

Oesophageal or gastric performation is an ever-present risk of gastric lavage even without ingestion of corrosives but fortunately it is rare. In this centre it has only happened once in the last 5000 washouts. Obviously attention to the design, flexibility and consistency of lavage tubes can help reduce the risk but avoidance of undue force by the operator and some degree of cooperation from the patient are also important. The latter is probably the most variable factor but in general it is probably true to say that the more uncooperative the patient, the less lavage is needed. A brief period of observation may resolve the dilemma. If the patient deteriorates it may be possible to empty the stomach with less resistance. On the other hand, if the patient improves during that time, lavage is probably superfluous.

\section{Does gastric lavage really recover poison?}

Much of the disenchantment with gastric lavage stems from apparently poor drug recovery for considerable effort expended. Inspection of the gastric contents of most patients reveals little evidence of tablet or capsule fragments and while this is an unacceptable method of assessing the value of the procedure, the general impression that it is often unrewarding is supported by the few studies in which the drug content of gastric aspirate and lavage fluid has been analysed. Allan (1961) obtained an average of $117 \mathrm{mg}$ of barbiturate from 68 patients but in $21(31 \%)$ none was recovered. Similarly Matthew et al. (1966) recovered more than $200 \mathrm{mg}$ of barbiturate in only 25 of 148 cases 
(17\%). The best result obtained by Allan was $1.1 \mathrm{~g}$ and by Matthew et al. $5.59 \mathrm{~g}$, but i the latter series the recovery exceeded $2 \mathrm{~g}$ (about 10 therapeutic doses of most barbiturate hypnotics) in only two patients. More recent experience with barbiturate merely serves to confirm that gastric lavage only retrieves large amounts in a minority of those treated. Comstock et al. (1981) recovered more than two therapeutic doses i $13.9 \%$ of poisonings with short-acting barbiturates and $22.7 \%$ with phenobarbitoneg However, only five patients surrendered between 11 and 20 therapeutic doses.

These figures beg the question how much drug recovery is justification for the procedure? Matthew et al. (1966) considered more than $200 \mathrm{mg}$ of a barbiturate was enough while Comstock et al. (1981) regarded two therapeutic doses as success. But b any standard these are small amounts and whether their recovery in borderline cases would weight the scales in favour of survival or reduced morbidity by the time patientis reach hospital must be open to serious doubt. In my experience, patients who die i⿱ hospital from poisoning do so because the expected toxicity of the poison cannot b] ameliorated (e.g. paraquat, or late paracetamol poisoning) or because they have sustained serious or irreversible damage (ARDS, cerebral oedema, cardiorespirator arrest) before reaching hospital. They do not die because they have not had theif stomachs emptied and only rarely do they go on absorbing sufficient drug (with or without gastric emptying) that they become so severely intoxicated after admission that they die.

Lavage was more rewarding in amitriptyline overdosage with $53 \%$ and $33 \%$ of 15 patients yielding more than two or 10 therapeutic doses respectively. The correspond ing figures in 29 cases of diazepam overdosage were $52 \%$ and $14 \%$ (Comstock et $\mathrm{g} l$. 1981). The situation in respect of salicylates is also better with 11 out of 22 (50 yielding more than $400 \mathrm{mg}$ including two about $5 \mathrm{~g}$ and one as much as $20.3 \mathrm{~g}$ (Matthe et al., 1966).

\section{Patient selection}

There are a variety of reasons why gastric lavage should be unproductive, but undoubtedly the main one is patient selection. Many doctors would like to have clear:cut rules about when to attempt gastric emptying but this is unrealistic for severa reasons. Clearly the toxicity of potential 'poisons' varies extremely widely and othei factors such as the number of poisons taken, the amounts involved, the time interval since ingestion, their pharmacological and toxicological effects (particularly on the gut and pre-existing abnormalities of structure and function of the upper gastrointestinat tract must be considered. While some science can be brought to bear on making decision, it ultimately becomes a matter of judgement and experience, attributes which are understandably in short supply amongst the junior members of medical teams of whom these burdens usually fall. Each case must be judged on its merits and the process్ must be conscious and thoughtful if patients are not to be subjected needlessly to distressing and potentially hazardous treatment. Gastric lavage must never become spinal reflex when faced with poisoning by ingestion, and it is totally unacceptable tha decisions be left to nurses.

The complexities, assuming they are appreciated in the first place, are sufficient to 
deter thought in many cases and precipitate action aimed at erring on the safe side with the inevitable consequence that too many patients are washed out unnecessarily. A recent study in Newcastle (Blake \& Bramble, 1978) found that $77 \%$ of patients with self-poisoning were subjected to gastric lavage-an extraordinarily high figure when compared with $44 \%$ in Edinburgh (Proudfoot) and 34\% in Houston, USA (Comstock et al., 1981). The fact that this survey also showed that the proportion of patients with benzodiazepine overdosage who were washed out was the same as those who had taken tricyclic antidepressants indicates a failure to appreciate the wide disparity in the toxicity of these two groups of drugs, far less the almost benign nature of benzodiazepines in excess. Indeed, the latter is such that I would seriously question whether ingestion of less than 30 therapeutic doses, even in conjunction with alcohol, is justification for lavage and even that number is arbitrary and arguably too low.

The time interval from ingestion and the patient's clinical state are also important factors in determining the amount of drug recovered. Allan (1961) obtained most when lavage was performed within $3 \mathrm{~h}$ of barbiturate overdosage while Matthew et al. (1966) recovered more than $200 \mathrm{mg}$ barbiturate in 24 out of $65(37 \%)$ lavaged within $4 \mathrm{~h}$ compared with only 1 out of $65(1.5 \%)$ treated later. The studies of Comstock et al. (1981) confirmed these observations and suggest that the period for significant drug recovery may be less than $2 \mathrm{~h}$. However, exceptions were not infrequent. The $2 \mathrm{~h}$ limit was least important in amitriptyline overdosage where the anticholinergic action of the drug would be expected to delay gastric emptying.

It is also generally agreed (Matthew et al., 1966; Comstock et al., 1981) that the interval since ingestion is less critical in patients who are deeply unconscious as a result of an overdose. One would expect that they had ingested larger than average quantities in the first place but the possibility that gut activity is depressed may also be important.

\section{Technical considerations}

The technical aspects of gastric lavage which require attention if maximum drug recovery is to be achieved should be well known (Matthew \& Lawson, 1979). Briefly, the patient should lie head-down in the left lateral position and the stomach tube must be of adequate size. The Jacques tube (English size 30) which has been recommended for years has a sufficiently large diameter and side-openings to allow the passage of food as well as tablet and capsule fragments. It is therefore less likely to become blocked. Tepid tap water is suitable for lavage in the great majority of cases and while additives are occasionally recommended they are seldom of critical importance and the procedure should not be delayed if they are not immediately available. The volume of lavage fluid is more important than additives. Again there is no fixed rule but aliquots of $300 \mathrm{ml}$ (for adults) should be used until the returning fluid is clear.

A number of variations of lavage technique have been recommended. Bartecchi (1974) suggests (on the basis of one case without drug analysis) that massaging the left upper abdominal quadrant during lavage may increase drug recovery by dislodging fragments stuck in the depths of mucosal folds. While this seems sensible and harmless enough, its value has not been assessed. Sharman et al. (1975) recommend following lavage by hourly aspiration of gastric contents through a naso-gastric tube. The bulk of 
additional drug recovered by this manoeuvre was usually obtained within the first 4 but only in one case out of 20 did the combined aspirates contain more than a sing therapeutic dose. It is therefore impossible to accept the authors' conclusion that th extension of gastric lavage was of 'considerable value'. Other variations (Miller, 1980 including the use of double-lumen naso-gastric tubes, continuous gastric suction an normal saline instead of water (both in woefully inadequate volumes) are suspect i⿱ principle and of no proven value.

The final technical question concerns who should carry out the procedure. Although nurses undoubtedly have much greater experience of passing naso-gastric tubes than doctors, it is doubtful if they are any more familiar with stomach tubes and lavagé. Ultimately it is probably of little importance who does it provided that the objectives are clearly understood, training is adequate and responsibility for complications is accepted.

\section{Psychological aspects of gastric lavage}

In theory, gastric lavage is easy but in practice it can be a traumatic experience fo f patient and operator alike. The psychological distress it causes is no doubt part of the reason why paediatricians have abandoned stomach tubes in favour of emetics. The effect of lavage on adults can also be dramatic. One psychiatrist questioning wh overdose patients admitted to a poisoning treatment centre were less likely to tak another overdose suggested that the consummatory nature of the drama was not only ifi the pill swallowing but also in the gastric lavage (Oswald, 1972). 'The Jacques tubogit thrust-red, lubricated, and of quite daunting diameter-deep into the primal orifer

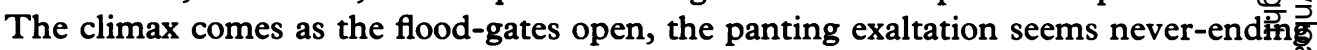
till, at long last and before the small gathering, the dripping instrument is withdrawn. new peace now dawns, life and hope are reborn. Those who survive never forget $\stackrel{9}{\rightarrow}$ Behind this Freudian concept is the serious question of whether gastric lavage has $\vec{a}$ deterrent effect. However the facts leave no doubt that the 'memorable experience' does not reduce the likelihood of future overdoses (Kennedy, 1972).

The effects on staff should not be forgotten either. Adults who poison themselves often present at unsocial hours and all too frequently are disinhibited by drugs and/o alcohol to the extent that they are abusive, uncooperative, perhaps violent and, io general, less than appreciative of the attentions of the caring team. To even pass 8 stomach tube in such circumstances requires tact, cajoling and patience at a time whe these virtues may be strained. Satisfactory completion of the procedure is equallg demanding. Add to these the vocal accompaniment, tubes blocking, soiling of clothes and body with gastric contents and the inherent dangers and it is easy to appreciate why. many would be glad to see the abandonment of gastric lavage. Human nature beinf what it is, such situations do not always bring out the best in us but one hopes that gastric lavage will always be carried out to retrieve drug and not for punitive reasons

\section{CONCLUSION}

Abandon gastric lavage in the accident and emergency department? - no, and probably never. By all means let us acknowledge its numerous limitations but not forget that i伭 
some cases, albeit a small minority, it can recover substantial quantities of drug thus reducing morbidity and possibly even mortality. Instead, let us try to determine how we can better select the patients who are likely to benefit so that fewer with little or harmless drug in their stomachs will be spared distressing treatment. Let us also try to bring a more thoughtful approach to the use of gastric lavage. Considerably more research is required and should be easier than 20 years ago because of the explosion in knowledge about drug actions, pharmacokinetics and disposal, not to mention the development of accurate methods of analysis. But research must be carried out in the clinical context-to use models such as bags of infusion fluids to represent the stomach only brings clinical toxicology into disrepute. The expertise and technology are available. What is lacking is thought and a will to resolve the problems.

\section{REFERENCES}

Allan B. C. (1961) The role of gastric lavage in the treatment of patients suffering from barbiturate overdose. Medical Fournal of Australia 2, 513-4.

Bartecchi C. E. (1974) A modification of gastric lavage technique. Fournal of the American College of Emergency Physicians 3, 304-5.

Blake D. R. \& Bramble M. G. (1978) Is there excessive use of gastric lavage in the treatment of self-poisoning? Lancet 2, $1362-4$.

Bush F. (1822) On the common syringe with a flexible tube, as applicable to the removal of opium and other poisons from the stomach. London Medical and Physical fournal 48, 218-20.

Comstock E. G., Faulkner T. P., Boisaubin E. V., Olson D. A. \& Comstock B. S. (1981) Studies on the efficacy of gastric lavage as practised in a large metropolitan hospital. Clinical Toxicology 18, 581-97.

Haddad L. M. \& Winchester J. F. (1983) In Clinical Management of Poisoning and Drug Overdose. W. B. Saunders Company.

Jukes E. (1822) New means of extracting opium \& c from the stomach. London Medical and Physical fournal 48, 384-9.

Kennedy P. (1972) Poisoning treatment centres. British Medical fournal 4, 670.

Lawson A. A. H., Proudfoot A. T., Brown S. S., MacDonald R. H., Fraser A. G., Cameron J. C. \& Matthew H. (1969) Forced diuresis in the treatment of acute salicylate poisoning in adults. Quarterly fournal of Medicine 38, 31-48.

Matthew H., Mackintosh T. F., Tompsett S. L. \& Cameron J. C. (1966) Gastric aspiration and lavage in acute poisoning. British Medical fournal 1, 1333-7.

Matthew H., Lawson A. A. H. (1979) In Treatment of Common Acute Poisonings, 4E. Churchill Livingston.

Miller M. (1980) Alternate methods for gastric lavage. Fournal of Emergency Nursing 6, 54.

Oswald I. (1972) Poisoning treatment centres. British Medical fournal 4, 430.

Proudfoot A. T., Park, J. (1978) Changing pattern of drugs used for self-poisoning. British Medical fournal 1, 90-3.

Proudfoot A. T. (1982) In Diagnosis and Management of Acute Poisoning. Blackwell Scientific Publications.

Proudfoot A. T. Unpublished data.

Sharman J. R., Cretney M. J., Scott R. S. \& Janus E. D. (1975) Drug overdoses: is one stomach washing enough? New Zealand Medical fournal 81, 195-7.

Schwartz H. S. (1976) Acute meprobornate poisoning with gastrotomy and removal of a drug-containing mass. New England fournal of Medicine 295, 1177-8.

Vale J. A. \& Meredith T. J. (1981) In Poisoning, Diagnosis and Treatment. Update Books.

Victor L. B., Gordon E. L. \& Greendyke R. M. (1968) Therapeutic implications of autopsy findings in acute barbiturate intoxication. New York State fournal of Medicine 68, 2090-2. 